\title{
ALCOHOL-RELATED LIVER DISEASES
}

\author{
Jiři Ehrmann, Ondřej Urban, Pavol Dvoran \\ Department of Internal Medicine II - Gastroenterology and Geriatrics, Faculty of Medicine, Palacký University Olomouc and University Hospital, \\ Olomouc, Czech Republic
}

\section{SUMMARY}

This educational article/narrative review reflects the European Association for the Study of the Liver (EASL) clinical practice guidelines: management of alcohol-related liver disease. An important change contrasting with the 2012 guidelines is the new terminology of alcohol-related liver diseases. Another important outcome is the strong emphasis on prevention of alcohol use disorder which may be performed at all stages of public health care.

Key words: ALD history, epidemiology, diagnostic methods, ACLF, liver transplantation

Address for correspondence: J. Ehrmann, Department of Internal Medicine II - Gastroenterology and Geriatrics, Faculty of Medicine, Palacký University Olomouc and University Hospital, I. P. Pavlova 6, 77520 Olomouc, Czech Republic. E-mail: ehrmanj@fnol.cz

https://doi.org/10.21101/cejph.a5999

\section{INTRODUCTION}

Medical authorities and laymen alike have considered, or even still consider, extensive alcohol drinking to be primarily associated with liver damage. After all, one third or even a half of liver diseases originate due to alcohol abuse, and 'hardening of the liver' is the feared liver disease of excessive drinkers. However, since the first scientific interest in the influence of alcohol on health attention was also paid to ailments other than those of the liver, irrespective of psychosocial aspects, as follows from constituent articles of the Journal of Studies on Alcohol and Drugs supplement which was written on the 75-year anniversary of the journal's origin (1). In fact, interest in alcohol-related liver disease increased in connection with a hot medicine topic - non-alcoholic fatty liver disease (NAFLD). Even though the aetiopathogenesis of these diseases is different, histological and clinical pictures are similar, including unfavourable consequences. This was one of the reasons why the European Association for the Study of the Liver (EASL) worked up and issued guidelines for the management of the alcohol-related liver diseases - the EASL Clinical Practice Guidelines: Management of Alcohol-Related Liver Disease - and published them in the Journal of Hepatology (2). In this article, these guidelines are taken into consideration.

\section{History}

The association between alcohol drinking and liver cirrhosis development was first described by Matthew Bailie in 1793. Later, Thomas Addison described fatty liver degeneration - steatosis - as a consequence of excessive alcohol consumption. In 1819 René Théophile Laennec coined the term liver cirrhosis. Today the terms Laennec's cirrhosis or portal cirrhosis are not used, but formerly they were used to name cirrhosis of alcoholic or viral origin. In 1842 William Bowman determined histological criteria for liver steatosis, which is the most common liver disease related to alcohol
(3). Scientifically supported knowledge about the unfavourable effects of alcohol on human health has its origin in the Department of Applicated Physiology, Yale University (USA). In 1940 this institution began to publish the Quarterly Journal of Studies on Alcohol, today named the Journal of Studies on Alcohol and Drugs. In the first number of this journal, K. M. Jellinek and N. Joliffe presented an analysis of all works about alcohol effects on health that had previously been published. Even though direct alcohol damage of the liver was supposed, the nutrition status, addiction development, genetic and other influences were considered to be the key factors in the aetiopathogenesis of liver damage. The key study, which proved the causal importance of alcohol in alcoholic liver fibrosis, was published by Mak et al. The authors observed lipocyte (Ito's cells) ultrastructure through baboon liver autopsies, and its change into fibrosis caused by a many-year diet in which alcohol took up half of the energetic input, against a second control group in which alcohol was not present. This work was announced to be one of the milestone publications of the 20th century by the Journal of Hepatology (EASL press organ) $(4,5)$.

\section{Terminology}

In recent years, alcoholic liver disease (ALD) terminology has been much discussed. Psychiatricians and psychologists object that existing terminology may stigmatise patients who suffer from alcohol overuse, but are not addicted, i.e they are not alcoholics. The recommended new terminology is shown in Table 1. It is not an academic formal terminology innovation. The issue is that it is a recommendation with an impact on the strategy of management of alcohol-related liver damage.

\section{Epidemiology of ALD}

ALD develops in consumers of more than two drinks per day (EASL recommends considering one drink as containing $10 \mathrm{~g}$ of 
Table 1. Terminology of alcoholic liver diseases (2)

\begin{tabular}{|l|l|l|}
\hline Previous term & Current term & Abbreviation \\
\hline Alcoholic & Alcohol use disorder & AUD \\
\hline Alcoholic liver disease & $\begin{array}{l}\text { Alcohol-related liver } \\
\text { disease }\end{array}$ & ALD \\
\hline Alcoholic cirrhosis & $\begin{array}{l}\text { Cirrhosis due to alcohol- } \\
\text { related liver disease }\end{array}$ & ALD cirrhosis \\
\hline $\begin{array}{l}\text { Alcoholic steatohepatitis } \\
\text { (histologically-defined } \\
\text { lesion) }\end{array}$ & $\begin{array}{l}\text { Steatohepatitis due to } \\
\text { ALD }\end{array}$ & ASH \\
\hline Alcoholic fibrosis & Fibrosis due to ALD & ALD fibrosis \\
\hline Alcoholic hepatitis & Alcoholic hepatitis* & AH \\
\hline
\end{tabular}

*However, at this point the term alcoholic hepatitis has become too standardised to change but may be reviewed in future guidelines.

alcohol). Most of these consumers suffer from greater or smaller hepatomegaly due to steatosis, a benign state which is reversible after 6-8 weeks of abstinence. However, steatohepatitis develops in $25-35 \%$ of these consumers, and fibrosis and cirrhosis develop in $8-20 \%$. The reason for this is still unknown. Liver cirrhosis is proved necroptically in only $18 \%$ of dissected patients with an evidence of heavy alcohol consumption during their lifetime, bioptically in only $17-31 \%$ (6). Nevertheless, the relative risk of cirrhosis development increases rapidly in dependence on the amount and total duration of alcohol consumption. Comparing the consumption of $20 \mathrm{~g}$ of alcohol per day over a period of 10-12 years, consumption of 40-60 g increases the risk 6 times and consumption of 60-80 g per day even 14 times. According to Lelbach, liver cirrhosis develops in $50 \%$ of daily consumers of $210 \mathrm{~g}$ of alcohol per 22 years and in $80 \%$ in those, who consume the same amount for 33 years (7). Generally, it is stated that cirrhosis is a certainty when drinking 180-200 g daily for 25 years. In women, the amounts are halved in most of the studies. On the other hand, abstinence improves the course of existing liver cirrhosis.

According to the Global Burden of Disease (GDB) study, in 2010 more than a million deaths due to liver cirrhosis were registered, which is $2.0 \%$ of all deaths. Of that, nearly $48 \%$ of cases were due to alcoholic liver cirrhosis. In 31 milion people liver cirrhosis was the cause of Disability Adjusted Life Years (DALY), which is an aggregated parameter denoting the lost years due to death from illness; alcoholic liver cirrhosis constituted $47 \%$ of this. Hepatocelular carcinoma in the liver cirrhosis terrain caused $80,600$ deaths in 2010 ( $0.15 \%$ of all deaths $)$, and also caused DALY in $2,142,000$ persons $(0.09$ of all DALY) $(8,9)$.

In epidemiological studies other factors should be considered, such as regularity or spasmodic character of drinking (so called binge drinking) and consumption of alcohol on an empty stomach or after eating. From the point of view of liver damage, episodic drinking is less dangerous than drinking lesser amounts of alcohol daily, as the liver manages to regenerate. Alcohol should not be drunk at least 2 days per week. Drinking when eating is also more favourable than drinking between meals. Liver damage is not related to the type of drink, but only to amount of alcohol in the drink (10). Even though we have quite precise information about alcohol cirrhosis prevalence and morbidity due to cirrhosis by rate of alcohol consumption, we cannot say the same about steatosis or mild or moderate fibrosis. It is estimated that about $50 \%$ of all liver cirrhosis are caused by alcohol, but there are many geographical differences (e.g. prevailing religion). In Islamic countries cirrhosis rates are only $10 \%$, in France $90 \%$. In the Czech Republic about $30 \%$ of liver cirrhosis is caused by alcohol with a greater proportion evident in the South Moravian region.

\section{Diagnostic methods of ALD}

\section{Liver Biopsy}

Liver biopsy is important for definitive diagnosis of ALD, exact staging and for exluding other causes of liver disease. Approximately $20 \%$ of patients with a history of inappropriate alcohol consumption and abnormal liver function tests are found to have co-existing aetiology of their liver disease. For this reason, liver biopsy is performed in 2nd or 3rd phases of clinical trials concerning ALD (2). Nowadays, ALD is diagnosed on the grounds of clinical picture, laboratory findings and non-invasive methods of investigation. Therefore, biopsy is generally not necessary for ALD diagnosis with a view to the $2 \%$ risk of complications - including fatal outcomes $(11,12)$.

In 1980, when J. Ludwig et al. described non-alcoholic steatohepatitis (NASH) on the grounds of histological/clinical findings as a new separate nosological unit (13), they queried the clinical differentiation between NASH and alcoholic steatohepatitis (ASH). The histological picture of both is very similar, although an experienced pathologist may detect some differences.

\section{Laboratory Tests of Alcohol Consumption}

Laboratory tests for ALD are basically not very different from tests for hepatopathies of other aetiology. However, in the event of suspected alcohol use disorder (AUD), laboratory tests of alcohol consumption must be performed. We can divide them into direct and indirect methods $(2,14)$.

\section{Indirect Methods}

Serum activity of GGT (gamaglutamyltranspherase) determination reflects chronic alcohol abuse with $42-86 \%$ sensitivity and $40-84 \%$ specificity. GGT production in hepatocytes may be induced by many other xenobiotics too. However, GGT serum activity increases in the case of cholestasis, which limits the clinical interpretation of this test.

Serum activity of AST (aspartate aminotranspherase) determination reflects chronic excessive alcohol abuse with 43-68\% sensitivity and $56-93 \%$ specificity. The value can be increased in case of acute hepatitis of variable aetiology and in case of muscle damage (myocardial infarction, rhabdomyolysis).

Serum activity of ALT (alaninaminotranspherase) determination reflects chronic excessive alcohol abuse with 30-50\% sensitivity and $51-92 \%$ specificity. The values may be influenced (increased) by any acute or chronic liver disease.

Determination of $M C V$ (mean corpuscular volume) reflects the chronic excessive alcohol abuse with $24-74 \%$ sensitivity and $70-98 \%$ specificity. The values may be influenced (increased) by vitamin B12 and folic acid deficiency and by some haematological diseases.

Determination of \% of blood CDT (carbohydrate-deficient transferrin). CDT determination is one of the sensitive and spe- 
cific markers of alcohol abusus with 12-days half-time and cut-off $2.5-3 \%$. Sensitivity of this test is $24-45 \%$, specificity $70-98 \%$.

Other laboratory methods include concentration of triacylglycerols determination, folic acid determination, lactate determination, glycemia determination and others. However, they are not specific.

\section{Direct Methods}

Direct methods (2) include determination of breath ethanol. Sensitivity is $97 \%$, specificity $93 \%$. The detection window lasts 4-12 hours. Next, blood or also urine EtG (ethyl glucuronide) detection. This is a molecule modified by ethanol. Detection window lasts up to 80 hours, and up to 6 months when testing from hair. Sensitivity is $89 \%$, specificity $99 \%$.

\section{Spectrum of Liver Damage Due to Alcohol Abuse}

Includes simple liver steatosis, steatohepatitis, acute alcoholic hepatitis, fibrosis, cirrhosis, and hepatocellular carcinoma (HCC). Intensive studies are focused on the issue of the amount of alcohol, the duration of alcohol consumption and progression from one form into another, up to cirrhosis or HCC. For example, in a Danish study by Deleuran et al. (15), the progression of biopsy verified steatohepatitis into liver cirrhosis within 5 years was demonstrated in $16 \%$ of patients, whereas cases with simple steatosis progressed only in $6.9 \%$. This means that the type of liver damage is important for the prediction of progression, but abstinence is necessary in the case of simple steatosis as well. As follows from Lackner et al. (16), mortality due to ALD is $17 \%$ with timely diagnosis and $43 \%$ when the diagnosis is late. Regarding the conversion of liver fibrosis to cirrhosis there are risk factors other than alcohol. Especially smoking (17) in contrast to drinking coffee, which has the opposite effect (18). Other risk factors consist of sex, obesity, diabetes mellitus, HBV or HCV and HIV infections. At present, genetic factors such as PNPLA3 gene polymorphism are being intensively studied (2).

\section{Steatosis/Steatohepatitis Caused by ALD}

Liver steatosis - "fatty liver", fatty liver change is the most frequent and usually the first type of liver damage in patients with a history of alcohol abuse in $90 \%$ of cases. As a histological unit it is pathologically counted among reversible dystrophic changes in liver cells. Steatosis means that there is more than $5 \%$ of fat in hepatocytes. Histological changes are reversible. Absolute alcohol abstinence leads to regression. In contrast, continued alcohol consumption leads to steatohepatitis followed by liver fibrosis and cirrhosis. Steatohepatitis is not a benign illness; in addition to deposition of fat in hepatocytes, the histological picture is now characterised by an inflammatory infiltrate, regressive changes and Mallory-Denk's hyaline in hepatocytes. Subjective symptoms of these patients are diverse. Difficulties are often absent and hepatic examination is frequently indicated due to abnormal liver function tests, which are often performed for other reasons. Patients with a serious form of the disease suffer from pressure in right hypochondrium and gastrointestinal difficulties. Enlargement of the liver is present. Diagnosis is supported by laboratory tests, as described above. Ultrasonography is the primary diagnostic method although it cannot discern the origins of steatosis or steatohepatitis. Nowadays, mathematical models such as the SteatoTest are used in diagnostics of steatohepatitis. The most significant serum test for steatohepatitis is cytokeratin fragment $18 \mathrm{M} 65$ or $18 \mathrm{M} 30$ detection, even though it does not differentiate between ASH and NASH.

\section{Alcoholic Hepatitis}

Alcoholic hepatitis (AH) is a clinical syndrome characterized by newly developed icterus with or without other signs of liver decompensation in patients with abuse of alcohol after a few days alcoholic excess. The amount of consumed alcohol differs individually. Symptomatology also varies. Mild forms can be softened by alcohol abuse. Heavy forms of alcohol hepatitis can lead to hepatic failure. Severe forms of acute alcoholic hepatitis lead to hepatic failure. The patient is icteric, consciousness is influenced by encephalopathy and often also by alcohol-caused delirium. Bleeding may manifest due to thrombopenia and coagulopathy. There can also be signs of portal hypertension with ascites, esophageal varices and hepatorenal syndrome. The patient may have raised temperature, leucocytosis and acute abdomen have to be excluded (2). A very bad prognosis is connected with cholestatic course of alcoholic hepatitis (19). AH can also proceed chronically with liver fibrotization and liver cirrhosis development.

AH diagnosis is based on clinical and laboratory findings. Liver biopsy verifies the diagnosis and mainly excludes other possible origins of liver damage. It is mostly indicated in patients with prolonged course of the illness without cardinal improvement following 6-8 months abstention. AH prognosis is serious in case of severe course of the illness. For short-term survival estimation the so-called Maddrey score can be used. Values over 32 and hepatic encephalopathy indicate a very poor prognosis (6).

\section{Acute or Chronic Liver Failure and Alcoholic Hepa- titis}

Acute or Chronic Liver Failure (ACLF) is characterised by acute decompensation of chronic liver illness associated with organ failure and high short-term mortality. Up to $40-50 \%$ of ACLF has an unknown trigger mechanism, in other cases it is mainly due to acute alcohol abuse, sepsis or relapse of infectious hepatitis. Severe alcoholic hepatitis represents about $25 \%$ of ACLF (2). ACLF severity is conditioned by organ failure associated with a systemic inflammatory response of the organism. All cases of ACLF are indicated for treatment in an intensive care unit. For treatment, extracorporeal systems of liver support are indicated. Definitive treatment is represented by liver transplantation (20-23) which may be considered for all patients in the absence of contraindications.

\section{Liver Fibrosis and Cirrhosis Caused by ALD}

Significant long-term alcohol consumption leads to liver fibrotic changes development and their progression into liver cirrhosis. Liver fibrosis can be defined as a structural change of liver tissue with enormous fibrotic mass deposition due to defect of its synthesis and degradation with the predominance of production. Liver cirrhosis is defined as a chronic, usually progressive illness, which is histologically characterised by necrosis of hepatocytes, lobular structure transformation and defect of intrahepatic and 
intraacinous circulation. According to the size of nodes, cirrhosis can be defined in two types - macronodular and micronodular. In cases of alcoholic cirrhosis, micronodular cirrhosis with a node diameter of $0.3 \mathrm{~cm}$ is typical. The clinical picture of alcoholic cirrhosis is basically the same as in cirrhoses of other aetiologies. Patients are often asymptomatic for a long period. They first go to a doctor when first symptoms occur, with a picture of compensated or decompensated cirrhosis, i. e. with signs of portal hypertension, metabolic failure, cholestasis or HCC. Liver cirrhosis examination has changed in recent years, alcoholic cirrhosis included. Liver biopsy is still the gold standard in liver cirrhosis diagnostics. However, it is an invasive procedure, which can be burdened by many complications. Because of this, non-invasive methods have developed rapidly (24). Their advantage is the fact that they are repeatable. In addition, they are more acceptable than liver biopsy for patients. Liver fibrosis examination can be performed in two non-invasive ways: elastographic methods and serum markers plus liver fibrosis tests.

Liver fibrosis is a dynamic process. It is reversible. Causal therapy (abstinence) can prevent the fibrosis to progress into cirrhosis with development of portal hypertension. If abuse persists the cirrhosis comes with all the complications. Otherwise, absolute abstinence can better the progress of liver cirrhosis dramatically. Liver cirrhosis increases the risk of $\mathrm{HCC}$ too. We cannot prevent its development, but abstinence can decrease the risk. Regular ultrasonographical checks enable us to find the HCC in curable state - i.e. resection or liver transplantation.

\section{Liver Transplantation}

Considering the indications for liver transplantation (LT) presents two basic questions: firstly, is the patient's illness so severe that LT will give benefit and higher survival probability than another treatment? Secondly, will LT give the patient benefit, is the patient capable of surviving the operation and is the patient capable of consequent life-long compliance? One year survival following transplantation and graft is about $80-85 \%$. The results show improved survival, similar to cases of other aetiologies (25). Even though only a minority of patients with AUD fulfil the strict criteria for LT, the number of LT performed due to ALD has increased in the last two decades. Between 2004 and 2013 the number of LT for ALD in the USA increased up to $45 \%$. The number of suitable donors can be increased by a change of legislation widening the criteria for suitable donors, and investments into health care, infrastructure and education. Despite the fact that ALD is one of the three LT indications which are most common in Europe and the USA, it is still controversial from the public point of view (26). The general public and medical experts remain in doubt about the priority grade offered to ALD patients in transplantation programmes. This presents an example of when personal judgement can influence ethical performance in medicine. Public research using anonymous questionnaires show that the public and general practitioners prefer donation of organs to patients with hereditary or acquired diseases which are not behavioural in context. The main problem is finding patients who can be expected to abstain. This is why psychosocial judgement of long-term abstinence has to be performed. Psychiatric examination must be performed because addicton can be connected with personality disorders, depression, anxiety, abuse of other sub- stances and other psychiatric disorders. Multidisciplinary access is needed, assessing not only medical, but also psychological suitability for transplantation. The three main risk factors of alcohol relapse include the duration of abstinence prior to transplantation, poor social base and family history of alcoholism. Most of the programmes require a 6-month period of abstinence. Probably a 6-month period of abstinence may help some patients to treat their liver damage, so the LT would not be necessary. It may also identify patients incapable of abstaining for a long period following LT (26). Many studies have supported the validity of the 6-month criterion whilst noting the fact that many patients with relatively low risk of relapse have had to stay on the waiting list due to this given period. Despite frequent use of the 6-month criterion, the International Liver Transplant Society, United Network for Organ Sharing (UNOS) and clinical practical EASL guidelines for ALD have not formally recommended it $(25,26)$.

\section{Conflict of Interests}

None declared

\section{REFERENCES}

1. Pandida RJ. Introduction to alcohol studies: 75 years in review. J Stud Alcohol Drugs Suppl. 2014;75 Suppl 17:5-7.

2. European Association for the Study of the Liver. EASL clinical practice guidelines: management of alcohol-related liver disease. J Hepatol 2018;69(1):154-81.

3. Franken FH, Falk H. History of liver disease. In: McIntyre N, Benhamou JP, Bircher J, Rizzetto M, Rodes J, editors. Oxford textbook of clinical hepatology. Oxford: Oxford University Press; 1991. p. 1455-60.

4. Mak KM, Leo MA, Liber CS. Alcoholic liver injury in baboons: transformation of lipocytes to transitional cells. Gastroenterology 1984;87(1):188200

5. Bissell DM. Milestones in liver disease: the origin of hepatic myofibroblasts. J Hepatol. 2002;37(3):298-301.

6. Burton R, Sheron N. No level of alcohol consumption improves health. Lancet. 2018;392(10152):P987-8.

7. Ehrmann J, Schneiderka P, Ehrmann J Jr, Vítek L, Jirsa M, Zima T, et al. Liver injury subject to alcohol. In: Hůlek P, Urbánek P, et al. Hepatology. Prague: Grada Publishing; 2018. p. 336-62. (In Czech.)

8. World Health Organization. Global status report on alcohol. Geneva: WHO; 2011.

9. Rehm J, Anderson P, Manthey J, Shield KD, Struzzo P, Wojnar M, et al. Alcohol use disorders in primary health care: what do we know and where do we go? Alcohol Alcohol. 2016;51(4):422-7.

10. Ehrmann J Jr, Aiglová K. Liver biopsy. In: Hůlek P, Urbánek P, et al Hepatology. Prague: Grada Publishing; 2018. p. 121-39. (In Czech.)

11. Sherlock S, Dooley J. Alcohol and the liver. In: Sherlock S, Dooley J. Diseases of the liver and biliary system. Hradec Králové: Olga Čermáková; 2004. p. 381-98. (In Czech.)

12. Filingeri V, Francioso S, Sforza D, Santopaolo F, Oddi FM, Tisone G. A retrospective analysis of 1.011 percutaneous liver biopsies performed in patients with liver transplantation or liver disease: ultrasonography can reduce complications? Eur Rev Med Pharmacol Sci. 2016 Sep;20(17):360917.

13. Ludwig J, Viggiano TR, McGill DB, Oh BJ. Nonalcoholic steatohepatitis: Mayo Clinic experiences with a hitherto unnamed disease. Mayo Clin Proc. 1980 Jul;55(7):434-8.

14. Sakhuja P. Pathology of alcoholic liver disease, can it be differentiated from nonalcoholic steatohepatitis? World J Gastroenterol. 2014;20(44);1674-9.

15. Deleuran T, Grønbaek H, Vilstrup H, Jepsen P. Cirrhosis and mortality risk of biopsy-verified alcoholic puresteatosis and steatohepatitis: a nationwide registry-based study. Aliment Pharmacol Ther. 2012;35(11):1336-42.

16. Lackner C, Spindelboeck W, Haybaeck J, Douschan P, Rainer F, Terracciano L, et al. Histological parameters and alcohol abstinence determine long-term prognosis in patients with alcoholic liver disease. J Hepatol. 2017;66(3):610-8. 
17. Sandahl TD, Jepsen P, Thomsen KL, Vilstrup H. Incidence and mortality of alcoholic hepatitis in Denmark 1999-2008: a nationwide population based cohort study. J Hepatol. 2011;54(4):760-4.

18. Kennedy OJ, Roderick P, Buchanan R, Fallowfield JA, Hayes PC, Parkes J. Systematic review with meta-analysis: coffee consumption and the risk of cirrhosis. Aliment Pharmacol Ther. 2016;43(5):562-74.

19. Kjaergard LL, Liu J, Als-Nielsen B, Gluud C. Artificial and bioartificial support systems for acute and acute-on-chronic liver failure: a systematic review. JAMA. 2003;289(2):217-22.

20. Arroyo V, Moreau R, Kamath PS, Jalan R, Ginès P, Nevens F, et al. Acuteon-chronic liver failure in cirrhosis. Nat Rev Dis Primers. 2016;2:16041. doi: $10.1038 /$ nrdp. 2016.41 .

21. Heemann U, Treichel U, Loock J, Philipp T, Gerken G, Malago M, et al. Albumin dialysis in cirrhosis with superimposed acute liver injury: a prospective, controlled study. Hepatology. 2002;36(4 Pt 1):949-58.

22. Bañares R, Nevens F, Larsen FS, Jalan R, Albillos A, Dollinger M, et al. Extracorporeal albumin dialysis with the molecular adsorbent recirculating system in acute-on-chronic liver failure: the RELIEF trial. Hepatology. 2013;57(3):1153-62.
23. Finkenstedt A, Nachbaur K, Zoller H, Joannidis M, Pratschke J, Graziadei IW, et al. Acute-on-chronic liver failure: excellent outcomes after liver transplantation but high mortality on the wait list. Liver Transpl. 2013;19(8):879-86.

24. Friedman SL. Liver fibrosis - from bench to bedside. J Hepatol. 2003;38 Suppl 1:38-53.

25. Burra P, Senzolo M, Adam R, Delvart V, Karam V, Germani G, et al. Liver transplantation for alcoholic liver disease in Europe: a study from the ELTR (European Liver Transplant Registry). Am J Transplant. 2010;10(1):138-48.

26. Addolorato G, Bataller R, Burra P, DiMartini A, Graziadei I, Lucey MR, et al. Liver transplantation for alcoholic liver disease. Transplantation. 2016;100(5):981-7.

Received October 12, 2019 Accepted in revised form December 4, 2019 